relatively healthy population it is likely that some of the associations remained undetected; for instance, the prevalence of eating disorders was not high enough to examine DSP reliably.

\section{P061 CAN NON-PHARMACOLOGICAL INTERVENTIONS FOR NOCTURIA IMPROVE SLEEP OUTCOMES? A SYSTEMATIC REVIEW}

${ }^{1}$ Ian Kellar*, 'Asheligh Hyde, ${ }^{1}$ Amy Love, ${ }^{1}$ Charlotte Naylor, ${ }^{1}$ Amelia-Jayne Parkinson Naylor, ${ }^{2}$ Anna Weighall. ${ }^{1}$ School of Psychology, University of Leeds, Leeds, UK; ${ }^{2}$ School of Education, University of Sheffield, Sheffield, UK

\subsection{6/bmjresp-2019-bssconf.61}

Background Nocturia results in reduced sleep due to waking after sleep onset. Treatments for nocturia may result in improved sleep outcomes, but lack of evidence for long-term outcomes has brought pharmacological approaches to managing nocturia in to question. Behavioural approaches for nocturia management have not been assessed for their effects on sleep.

Objective To evaluate the evidence for non-pharmacological nocturia treatments on sleep outcomes in adults.

Search methods: Four databases (Medline, PsychInfo, Embase \& Web of Science) were searched, and relevant results were hand-searched for additional papers. Databases were last searched in November 2018. Selection criteria.

The population was adults; the interventions were nonpharmacological treatments for nocturia; outcomes were measures of sleep; study designs were restricted to randomised controlled trials. No limit was placed on comparison or year of publication. The publication type was restricted to journal articles in English.

Results Only 3 studies ( $\mathrm{N}=137$ ) were found to be relevant. No significant differences between intervention and control for nocturia outcomes was found in any study. There were mixed, but mostly null findings for objectively measured sleep outcomes. There was some evidence for subjective sleep outcomes.

Conclusion The small, underpowered studies that were found limit the conclusions that can be drawn from this evidence base. Given the loss of sleep quality associated with nocturia is implicated in the development of hypertension and type 2 diabetes, larger, appropriately powered trials should be undertaken.

\section{P062 NON-PHARMACOLOGICAL INTERVENTIONS FOR SLEEP PROBLEMS IN NEURO-TYPICAL CHILDREN: A SYSTEMATIC REVIEW}

${ }^{1}$ Clarice Turton*, ${ }^{1}$ Anna Hamilton, ${ }^{2}$ Anna Weighall, ${ }^{1}$ lan Kellar. ${ }^{1}$ School of Psychology, University of Leeds, Leeds, UK; ${ }^{2}$ School of Education, University of Sheffield, Sheffield, UK

10.1136/bmiresp-2019-bssconf.62

Context Sleep disturbances and disorders are common in children. Non-pharmacological interventions for such disorders are recommended by current guidelines. Recent reviews important in this area focus solely on children with neurodevelopmental disorders (Scantlebury et al., 2018). The current review seeks to synthesise the evidence for children in the the non-clinical population.
Objective To expand upon recent reviews by reviewing nonpharmacological interventions for sleep problems in neuro-typical children.

Data Sources Five electronic databases (MEDLINE, EMBASE, PsycINFO, CINAHL and Cochrane databases) were searched using search terms including and relating to 'Children', 'Sleep', 'Behavioural Interventions' and 'Randomised Control Trials'.

Study selection Randomised control trials using non-pharmacological interventions with a sleep outcome, for children and adolescents over five years old were included in the study.

Synthesis. Results were synthesised narratively in relation to intervention content, delivery and efficacy.

Conclusions The studies overall support the recommendation of using non-pharmacological interventions for sleep problems in children. The majority of research to date has investigated the efficacy of cognitive behavioural techniques and was found to be effective in a clinical setting. Future research should evaluate the feasibility and efficacy of these techniques applied on a wider scale and in home settings in order to reach more children.

\section{P063 SLEEP MATTERS UK: EXPLORING THE RELATIONSHIP BETWEEN SLEEP DIFFICULTIES AND QUALITY OF LIFE IN PRIMARY SCHOOL CHILDREN}

${ }^{1}$ Anna Weighall* ${ }^{2}$ Hannah Nash, ${ }^{2}$ lan Kellar. ${ }^{1}$ School of Education, University of Sheffield, Sheffield, UK; ${ }^{2}$ School of Psychology, University of Leeds, UK, Leeds, UK

\subsection{6/bmjresp-2019-bssconf.63}

Background Sleep efficiency is strongly related to academic performance and behavioural regulation across the lifespan (e. g., Fredriksen et al., 2004; Gruber et al., 2014). Lack of regular bedtimes has been associated with poorer cognitive abilities, including reading (Kelly et al., 2012). However, the relationship between sleep and wellbeing, and the scale of sleep problems in childhood is poorly understood.

Methods Data from a representative UK survey $(n=1,100)$ of parents with children aged 6-11 years old asked 60 questions (based upon well validated scales) including the Child Sleep Habit's Questionnaire (CSHQ) and Pediatric quality of life (QOL; measured by PedsQL) and family routines. Data were analysed using ANOVA, correlations and hierarchical linear regression.

Results The NHS recommends $\sim 10$ hours sleep in children of this age. Thirty-six percent of children achieved $<$ eight and $15.2 \%<$ seven hours - levels likely to impair daytime functioning, and development. Worryingly, sleep problems of clinical significance (CSHQ) were prevalent (over 90\%), Statistically significant relationships between poor sleep and lower QoL were found $(r=0.567, p=0.001)$. Shorter sleep duration was associated with a range of problems at school, eg. difficulties in paying attention in class, forgetting things, keeping up with school work and missing school because of illness (all $\mathrm{p}<0.001)$.

Conclusions and implications Sleep problems in UK primary school children are widespread, often at levels likely to affect daytime functioning and wellbeing, borne out by the association between poor sleep and poor QoL. Further research is needed including objective sleep measures, and longitudinal evidence in order for causal relationships to be 